\title{
Concept for a new approach to realize complex optical systems in high volume
}

Heinrich Grüger, Jens Knobbe, Tino Pügner, Michael Leuckefeld, Peter Reinig, et al.

Heinrich Grüger, Jens Knobbe, Tino Pügner, Michael Leuckefeld, Peter Reinig, Sebastian Meyer, "Concept for a new approach to realize complex optical systems in high volume," Proc. SPIE 10448, Optifab 2017, 1044803 (16 October 2017); doi: 10.1117/12.2279782

SPIE. Event: SPIE Optifab, 2017, Rochester, New York, United States 


\title{
Concept for a new approach to realize complex optical systems in high volume
}

\author{
Heinrich Grüger*, Jens Knobbe, Tino Pügner, Michael Leuckefeld, Peter Reinig, Sebastian Meyer \\ Fraunhofer IPMS, Maria Reiche Str. 2, 01109 Dresden Germany
}

\begin{abstract}
A groundbreaking new approach [1] for the fabrication of complex photonic systems, especially such with off-axis optics, has been invented based on planar mounting in combination with a novel folding approach.

Up to now volume production of photonic systems has been optimized for on-axis lens based optical systems. Chromatic aberration limits the usage or spectral range of these systems. Applying mirrors instead of lenses may help to suppress chromatic aberrations and wavelength depending absorption. The assembly of reflective optics, often in an off-axis configuration, is a complex process. So far most tools for volume production apply stacking of components in planar technology. Off-axis systems are typically assembled by more or less manually alignment of the components, which is not in favor for mass and low cost production of these systems.

The novel approach utilizers a planar substrate featuring preprocessed bending lines. A high accuracy tool for planar assembly places the components onto the substrate. Then the sides of the substrate are bent leading to a predefined three dimensional body. The off-axis optical path inside is generated automatically.

This concept is not limited to rectangular shapes but can also be applied to more complex systems, for example the so called "W-configuration" for a Czerny-Turner spectrometer.

First tests of the "bend and place assembly" have been performed successfully on a camera setup to prove the working principle.
\end{abstract}

Keywords: planar assembly, alignment, optical system, off axis, spectrometer, camera, multispectral, infrared

\section{INTRODUCTION}

Miniaturized optical systems have entered high volume production throughout the last years. Especially digital camera devices have reached a unit volume in the billion per year range. Assembly technologies for these on axis systems rely mostly on stacking optical components to produce the optical bench (fig. 1). The optical setup can be complex and may also include adaptive elements for focus and zoom.

*heinrich.grueger@ipms.fraunhofer.de; phone +49 3518823 155; fax +49 3518823 266; www.ipms.fraunhofer.de 


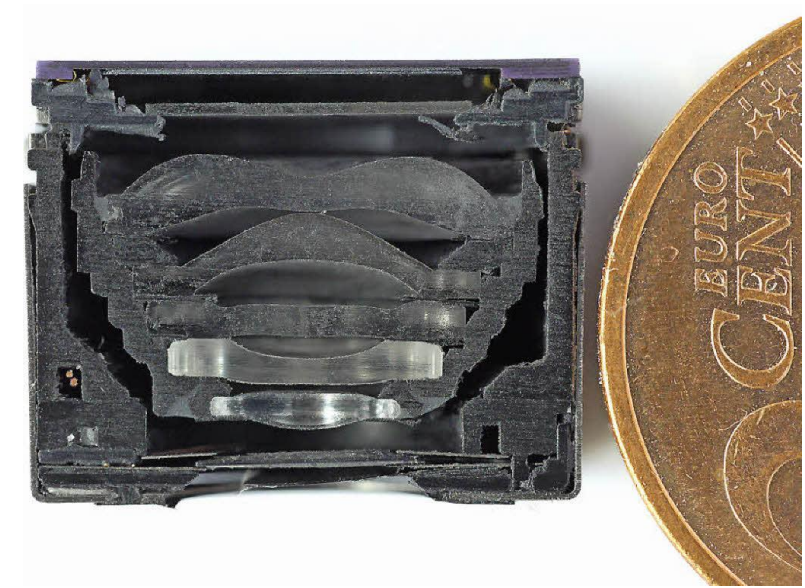

Figure 1. Photograph of a cross section through a camera module for mobile phone; image Carl Zeiss [2]

New applications for optical systems will arise in the coming years. From recent system developments it can clearly be concluded, that an extended spectral range reaching into the near infrared region (NIR, $780 \mathrm{~nm} \ldots 2500 \mathrm{~nm}$ ), mid infrared (MIR, $2.5 \mu \mathrm{m} \ldots 8 \mu \mathrm{m}$ ) or even thermal infrared $(8 \mu \mathrm{m} \ldots 15 \mu \mathrm{m})$ opens multiple new possibilities for the enduser. Infrared or broadband optical applications request more sophisticated or alternative kinds of optical systems to meet the demanding requirements. This will be a challenge for both developer and manufacturer of the optical systems. Affordable transparent materials for infrared which offer high volume production capabilities are limited. Consequently lens based systems face more restrictions than just chromatic aberration.

Reflective optics lends itself as an alternative to lens based systems. Large telescopes are prominent examples of such systems. The replacement of lenses through optical mirrors offers at least two significant advantages: Reflective materials to cover the range from UV to thermal IR are available and chromatic aberration is not an issue. But there are also some drawbacks which must be considered: Wide range spatial adaptive mirrors are not yet available, cost might become an issue and system designs lead to a choice between performance losses from central obscuration or complex off-axis optic designs. A prominent example for an off-axis optical system is the "Schiefspiegler" from Anton Kutter [3].

One major limitation of the "Schiefspiegler" is the image distortion. Today applying digital detectors and software reconstruction algorithms this limitation can be overcome. Image reconstruction is reaching sub-pixel accuracy [4]. In comparison to lens based systems the correction of chromatic aberration is much simpler and a reduced number of optical elements can be sufficient to achieve satisfying performance. Up to now the fabrication of sophisticated optical mirrors is less common. Thus the production of complex optical systems is more expensive with current assembly technologies like manual or semimanual 3D integration.

Other optical off-axis systems like spectrometers face the same situation. Unfortunately only a limited set of alternatives for spectral analysis based on on-axis designs is available. Spectral filters deposited directly on the detectors is one option, but detector arrays are required leading to increased size and cost, especially in the infrared region. Fabry-Perot interferometers as tunable filters are another possibility, but the free spectral range is limited. Furthermore these system have high requirements for the planarity of the moving mirror.

Summarizing it can be stated that reflective optics possess superior optical properties for optical systems. Complex optical systems based on reflective elements often lead to off-axis designs which have specific requirements for the alignment. Up to now the assembly processes available for these systems are not suitable for mass and low cost production.

If off-axis designs shall be competitive in the market for low cost and ultra-compact systems the assembly and alignment issues have to be solved. This is the starting point of our new approach. 


\section{PREVIOUS WORK}

Starting point of the previous developments were micro electro mechanical systems (MEMS) which have been used to miniaturize sensors and actuators for more than 25 years. Commercial MEMS sensors have been integrated to a very high level. Devices in a super small outline package are produced in very high volume and can be purchased for few dollars.

MEMS acting as actuators are a bit more complex. Optical MEMS or MOEMS (micro opto electro mechanical systems), for example scanner mirrors [5] almost always operate in combination with further components like light sources (LEDs, lasers), optics, electronics or optionally detectors. Some prominent example are laser projection [6], retina scanning [7], LiDAR or other measurement systems. More sophisticated MOEMS have been realized by the integration of a diffraction grating on the tiltable plate of a scanner device replacing the simple mirror [8]. Based on this a miniaturized MEMS based spectrometer [9] has been built and optimized for near infrared (NIR) applications.

Optical MEMS based systems share the approach to miniaturize components by means of micro systems technologies. In many examples the system miniaturization reached a level where further size reduction is limited by alignment issues. Some examples from our own developments are described here.

The first MEMS scanning grating based spectrometer featured a volume of $720 \mathrm{~cm}^{3}$ (Hiperscan SGS 1900, data sheet see www.hiperscan.com), which is small compared to classical table top equipment. It provided $10 \mathrm{~nm}$ resolution in a spectral range from $950 \mathrm{~nm}$ to $1900 \mathrm{~nm}$ and entered the raw materials testing market. The next step in miniaturized MEMS based spectrometers was the development of a hybrid integrated setup fabricated by stacking of functional substrates. Both slits and the tiltable grating were integrated into the MEMS chip. The so called "sugar cube" size prototype [10] with a size of $2.1 \mathrm{~cm}^{3}$ revealed both options and limits of further miniaturization.

In a different research work a hyper spectral imaging camera was developed. Using a similar MEMS chip the detector requirement has been reduced from a $2 \mathrm{D}$ imager to a simple 1D line. A demo system was realized based on a "Schiefspiegler" optical design and has been tested successfully [11]. Again alignment turned out to be a complex task limiting further size reduction which would be advantageous especially for mobile applications.

Furthermore work with "Schiefspiegler" optics was performed based on the assumption that one day adaptive mirror in chip scale might become available. An evaluation kit for 3x zoom was realized [12] showing performance of chromatic aberration free reflective optics that will become possible with a four mirror system, two of them must be adaptive with precisely controlled deformation and $500 \mu \mathrm{m}$ deflection for a $6 \mathrm{~mm}$ mirror diameter. Here already the idea of beam splitters and double set of detectors for separated spectral ranges has been discussed [13].

Recently a first prototype of a multispectral camera has been finished. This device is capable to operate in the VIS range $(380 \ldots 780 \mathrm{~nm})$ and in MID / thermal infrared $(5 \mu \mathrm{m} \ldots 12 \mu \mathrm{m})$. Two detectors share the same optics with a beam splitter. High resolution RGB images can be correlated to thermal information from the $1024 \mathrm{x} 768$ element bolometer detector. The system operated successfully and might be interesting for applications like remote sensing from unmanned aerial vehicles or drive assistance in automobiles but size and cost must be reduced dramatically. One again, assembly and alignment becomes a limiting factor for size and cost reduction.

\section{BASIC IDEA TO PLACE COMPONENTS AND BEND THE SUBSTRATE "PLACE AND BEND ASSEMBLY"}

From the examples of previous developments the need for an improved assembly and alignment of complex optical systems, especially off-axis designs can clearly be derived.

If a spectrometer can be miniaturized to a level that would enable integration into mobile phones and if production can reach a price level acceptable for the implementation, many applications would arise. Prominent examples are food analysis, health checkup or environmental monitoring. Simulations of the optical bench revealed that an ultra-compact size might be possible in combination with an adequate system performance. The major difficulty is the precise 
alignment of the two slits, both mirrors, the MEMS grating and an infrared detector in an ultra-small size Czerny Turner type spectrometer at reasonable cost.

A highly integrated MEMS device and stacking of functional substrates [14] could be one option. Nonetheless further size reduction could benefit from a completely new approach for an assembly process optimized for the high volume production of complex optical systems, especially off-axis reflective systems.

This completely new idea [1] for optical system integration is called "place and bend assembly" or "Faltmontage" in German. This could replace the manual or semimanual processes which are used today to assemble MEMS chips in 3D and align the optical path by microdrives or screws afterwards.

In the new approach a planar substrate is used which features preprocessed bending lines. The MEMS chip and other optical and electronic components are placed by conventional pick-and-place tools, which can be very accurate with state of the art equipment. Afterwards the substrate is bent and the complete housing is achieved by bending of the substrate along the preprocessed lines. In principle the bending approach is similar to paper boxes which parcel distributors provide but much smaller and much more precise. The optical path inside the system automatically adjusts through the structure of the substrate (see figure 2). The accuracy of the substrate together with the tolerances of the placement and bending processes must be precise enough to ensure that the final deviations can be tolerated by the optical design.

E. Fraunhofer

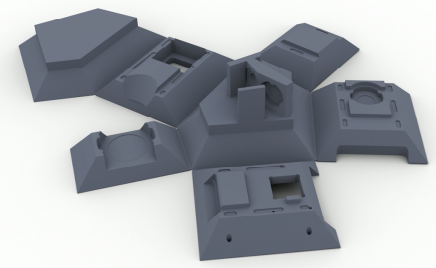

a)

E. Fraunhofer

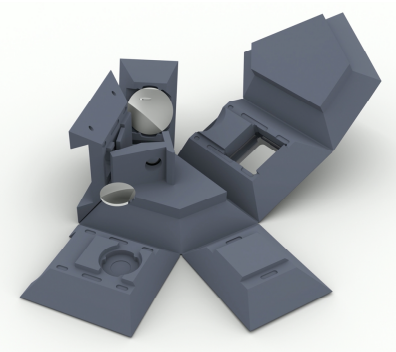

c)

E Fraunhofer

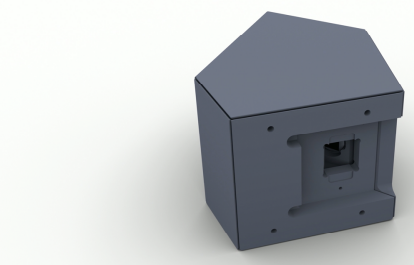

e)

Figure 2. Rendered images from the design of a simple camera applying "place and bend assembly".

From left to right / up to down: a) Planar substrate b) Planar substrate with components c) semi-folded system, open

d) almost closed e) final system f) optical path inside

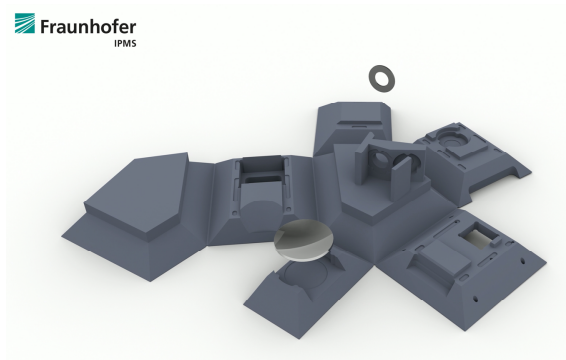

b)

$$
\text { Eraunhofer }
$$

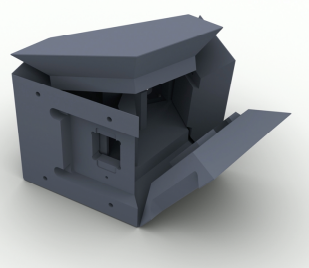

d)
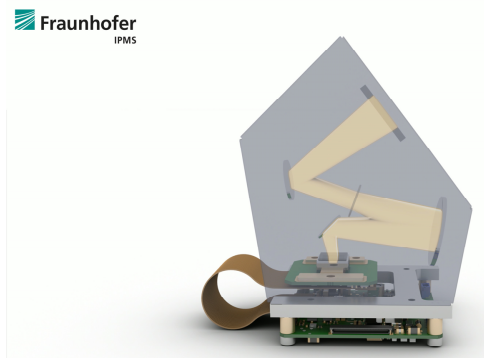

f) 


\section{SYSTEM REQUIREMENTS}

Based on our long term experience in the field of miniaturized MEMS based near infrared spectrometers and its relevance for future applications we have chosen the infrared spectrometer for an analysis of system requirements.

The "place and bend assembly" concept seems to be simple but the details can be quite complex. After finishing the assembly the optical path must meet the tolerance requirements of the optical design. Deviations originating from material or surface distortions as well as from process induced tolerance must be small. Off-axis designs considered here are the W-configuration of Czerny Turner spectrometer (fig. 3), but it could also be the zig-zag shaped optical path of a "Schiefspiegler" with its four mirrors or any other off-axis optical system.

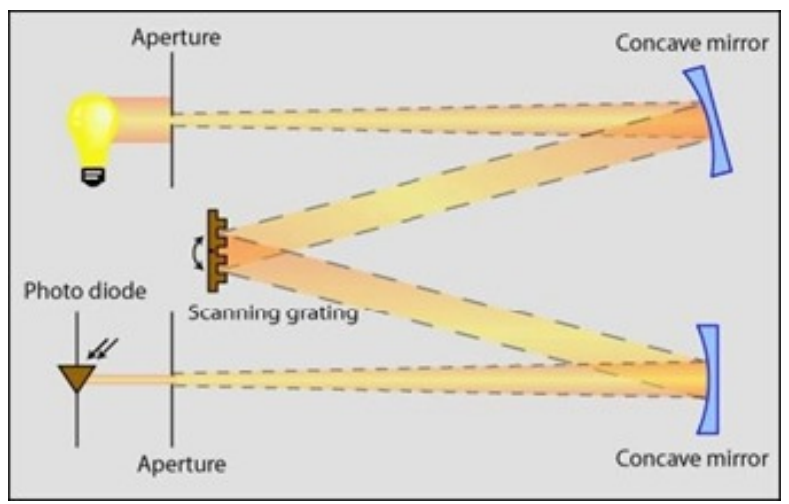

Figure 3. Typical optical path in W-configuration of Czerny Turner spectrometer

Starting point of the analysis are the requirements for the setup of the hybrid integrated MEMS spectrometer for near infrared (NIR) applications as shown in figure 4. The MEMS chip used for this design is not deflected in its rest position, thereof the spectrometer is operating in the first negative diffraction order. By placing both slits on the same side of the grating a folded design enables proper operation with a symmetric deflection of the non pre tilted grating with both slits and the grating in one plane generated by the chip surface. Both mirrors are integrated in one single part by ultraprecision machining. Due to the operation in the first negative diffraction order, a folded optical path is required inside for proper operation. The corresponding spectrometer parameters are collected in table 1.

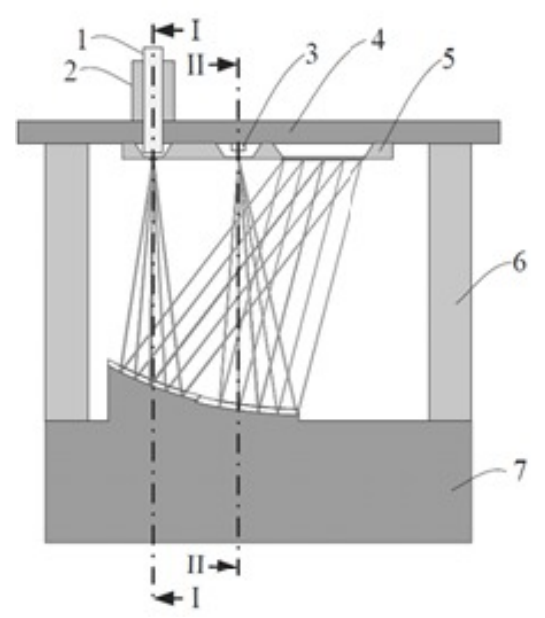

Figure 4. Folded optical path inside the miniaturized MEMS Czerny Turner spectrometer 
Table 1. Spectrometer parameters for the sugar cube size prototype.

\begin{tabular}{|l|l|l|}
\hline Parameter & Size & Dimension \\
\hline Focal length & 8.4 & $\mathrm{~mm}$ \\
\hline Entrance and exit slit width & 50 & $\mu \mathrm{m}$ \\
\hline Entrance and exit slit height & 250 & $\mu \mathrm{m}$ \\
\hline Grating length (square shape) & 3 & $\mathrm{~mm}$ \\
\hline Grating line density & 625 & $1 / \mathrm{mm}$ \\
\hline Corresponding grating d-spacing & 1600 & $\mathrm{~nm}$ \\
\hline Mirror size & $3 \times 3$ & $\mathrm{~mm} \times \mathrm{mm}$ \\
\hline
\end{tabular}

Up to now the assembly has been performed by stacking of functional substrates. The real deviations between design and device can be estimated from the prototypes. This can be correlated to the different assembly steps and the requirements can be derived. It was shown that the focal position must be within $\pm 10 \mu \mathrm{m}$ of the specified range or effects on the resolution become detectable.

Tolerable deviations for a spectrometer were derived from the sugar cube size setup. The alignment of both slits and the grating is assured by the MEMS process. The structures are realized by the same process step (front side dry etch), the layout is within the same mask. The tolerance inside this step is negligible. The grating on the scanner plate is etched by a different wet etch step. Mask alignment deviations could occur, but due to the quality of the equipment used today they will be much smaller than the grating d-spacing $(1600 \mathrm{~nm})$. Additionally the impact of deviations in both $\mathrm{x}$ and $\mathrm{y}$ direction is small. Angle misfits would be critical as they would deflect the light out of the spectrometer plane. Placement of alignment masks on diagonal edges of the reticle which has more than $10 \mathrm{~mm}$ width grants precise parallel alignment; $0.5 \mu \mathrm{m}$ lateral deviation would be much, thus the angle deviation is small $(\Delta \varphi=\arcsin (0.5 \mu \mathrm{m} / 10 \mathrm{~mm})<$ $\left.0.003^{\circ}\right)$.

The accuracy of the chip assembly is granted using alignment marks on the chip and on the substrate. As the chip is thin $(450 \mu \mathrm{m} \ldots 700 \mu \mathrm{m})$ the depth of focus is not a problem and the chip to substrate alignment is accurate within $0.5 \mu \mathrm{m} \ldots 2 \mu \mathrm{m}$ depending on size and effort for the alignment marks.

It can be concluded, that the chip alignment should reveal an accuracy of $1 \mu \mathrm{m} \ldots 2 \mu \mathrm{m}$. In our work a Finetech Fineplacer FEMTO ${ }^{\circ}$ has been used which meets this requirement.

The position of both mirrors has not been an alignment problem up to now as they are manufactured from the same substrate. If two separated mirrors should be used this could be an issue for future systems. Alignment marks will have to be placed on the edges of the mirrors.

The stacking step finishes the system assembly. The spacer substrate generates the distance between MEMS substrate with slits \& grating and mirror substrate. Spacer thickness deviations will lead to defocus, which is critical. Besides spacer thickness both adhesive layers may have an additional impact. Lateral deviations have only minor impact as long as they remain small and the mirrors are not illuminated out of the optical axis. In fact deviation $\Delta x, \Delta y$ of $20 \mu$ m should be tolerable. Rotation misfit leads to deflection of light out of the spectrometer plane which must be avoided. The final step includes a high topography thus less accuracy in lateral direction and the adhesive thickness thus tolerances of 10 $\mu \mathrm{m} \ldots 20 \mu \mathrm{m}$ may occur.

Summarizing the lateral placement of the MEMS chips and optical components must be accurate within in a $1 \ldots 2 \mu \mathrm{m}$ range for the new "place and bend assembly" approach. Alignment marks must be implemented in a way that ensures very low tilt of the components, especially for slits, gratings and other non-rotation symmetric components. The bending process which includes the position of the bending structures, deviations originating from the process itself and structural changes or misfit in the substrate must not exceed $10 \mu \mathrm{m} \ldots 20 \mu \mathrm{m}$. Finally focus positions are critical. Here $10 \mu \mathrm{m}$ must not be exceeded for a slit $50 \mu \mathrm{m}$ wide. Harder limits may occur for components with smaller structures, e.g. pixel elements of detector arrays. 


\section{CONCEPT DEVELOPMENT}

Miniaturization of complex optical setups is a major task to bring optical MEMS based systems into volume applications. The idea of stacking of functional substrates originally considered wafer stacking and related substrates but dicing issues become very critical for stacks with $8 \mathrm{~mm}$ in height or more. Despite this limitation, stacking has been successfully used to integrate a MEMS based spectrometer. However, searching a new approach to overcome limitations for volume production and using standard equipment with minor changes only lead to the new disruptive idea of bending a planar substrate.

A sophisticated planar assembly option has been available and process steps for both MEMS and system assembly were implemented successfully. Based on our experience with Czerny Turner type spectrometer development it is necessary to place optical components on two inner sides of a box facing each other. Here the first step of the idea and concept was to use a planar substrate with two preprocessed bending lines generating bottom and two sides facing each other, then place components precisely and fold the substrate. The next step was simply considering that mechanical stops would be helpful for alignment issues. Furthermore comparison to existing layout for folded boxes revealed potential to use one single substrate for the entire system housing and to have a very high accuracy within the assembly process and the substrate that grants the (auto-) alignment of the optical path.

Comparison of the requirements and data from planar assembly specifications and information about modern process technologies for 3D printing and plastic molding process capabilities revealed good chances for this approach.

Planar assembly tools are specified for accuracy in micrometer range and below. This was tested and confirmed for MEMS chips including large type of $9.6 \times 5.3 \mathrm{~mm}^{2}$ outline. Typically the deviations of the position range below $2 \mu \mathrm{m}$.

Regarding the substrate plastic technology providers have announced an accuracy for planar plastic substrates of $10 \mu \mathrm{m}$ or less. Proof is outstanding how far this remains valid for complex structures and lines with several millimeters in length. Acceptable thickness variation have to be confirmed as well as potential mechanical impact of the bending forces on the remaining substrate. Here also material selection may be a relevant issue.

In general 3D printing processes available today are attractive for rapid prototyping. The accuracy announced now by loan suppliers turned out to be less accurate than the $10 \mu \mathrm{m}$ announced for plastic processes. A selection of different materials can be used which is not limited to plastic material. Again a very important issue is the impact of the folding process on the substrate. The distortion from the folding must be negligible. Here material parameters, depth and shape of the bending lines will have to be investigated in detail.

Depending on the process selected some general ideas for the bending structures have been discussed so far. The advantages and limitations have to be discussed in much more details in the future. 

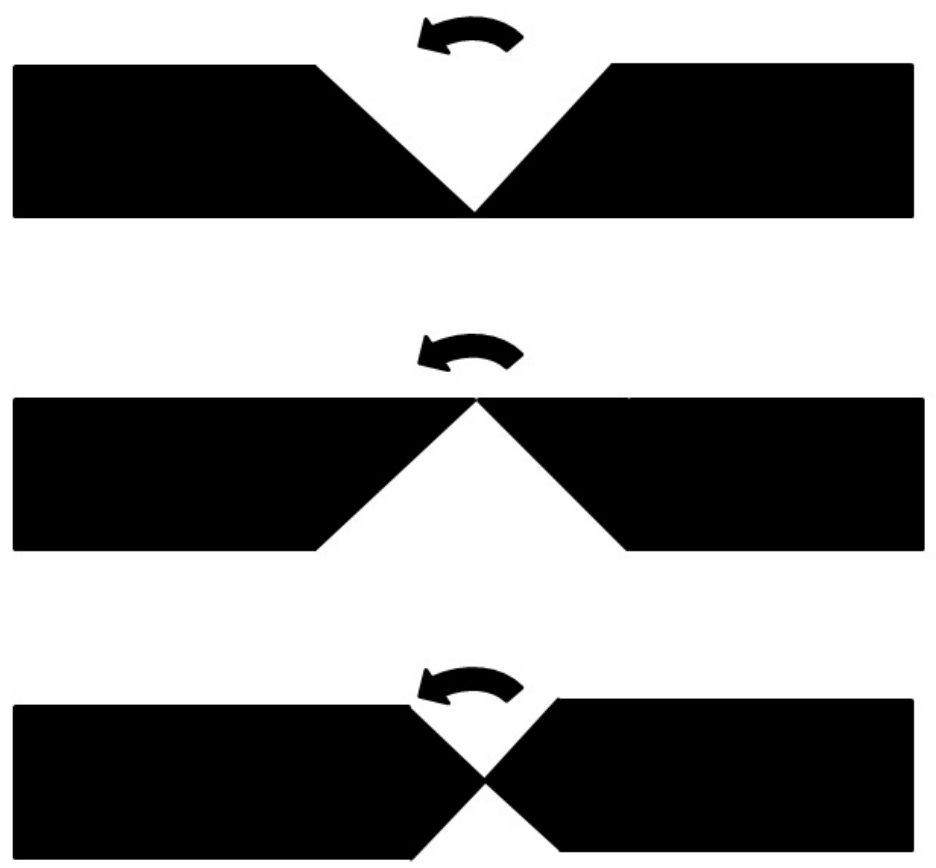

Figure 5. Examples of different options for bending structures in cross section

\section{PROOF OF CONCEPT}

A first step from concept to system development is proof of concept. A simple camera system has been selected which enables the evaluation of quality and deviations. This device uses a completely reflective approach based on a two spherical mirror design. Shortcomings like defocus, image distortion and misalignment become visible quite simply. A comparison between image quality extracted from simulation to images taken with the device will enable a performance analysis of the concept even if the image quality of a two spherical mirror design is limited by design.

For the proof of concept a camera has been realized from a 3D printed substrate (fig. 6, left). An entrance window, two spherical mirrors, an aperture stop and a detector array, all standard components from catalogue suppliers, have been assembled using planar technology (fig. 6, middle). Afterwards the substrate was bent and fixed (fig. 6 right). 


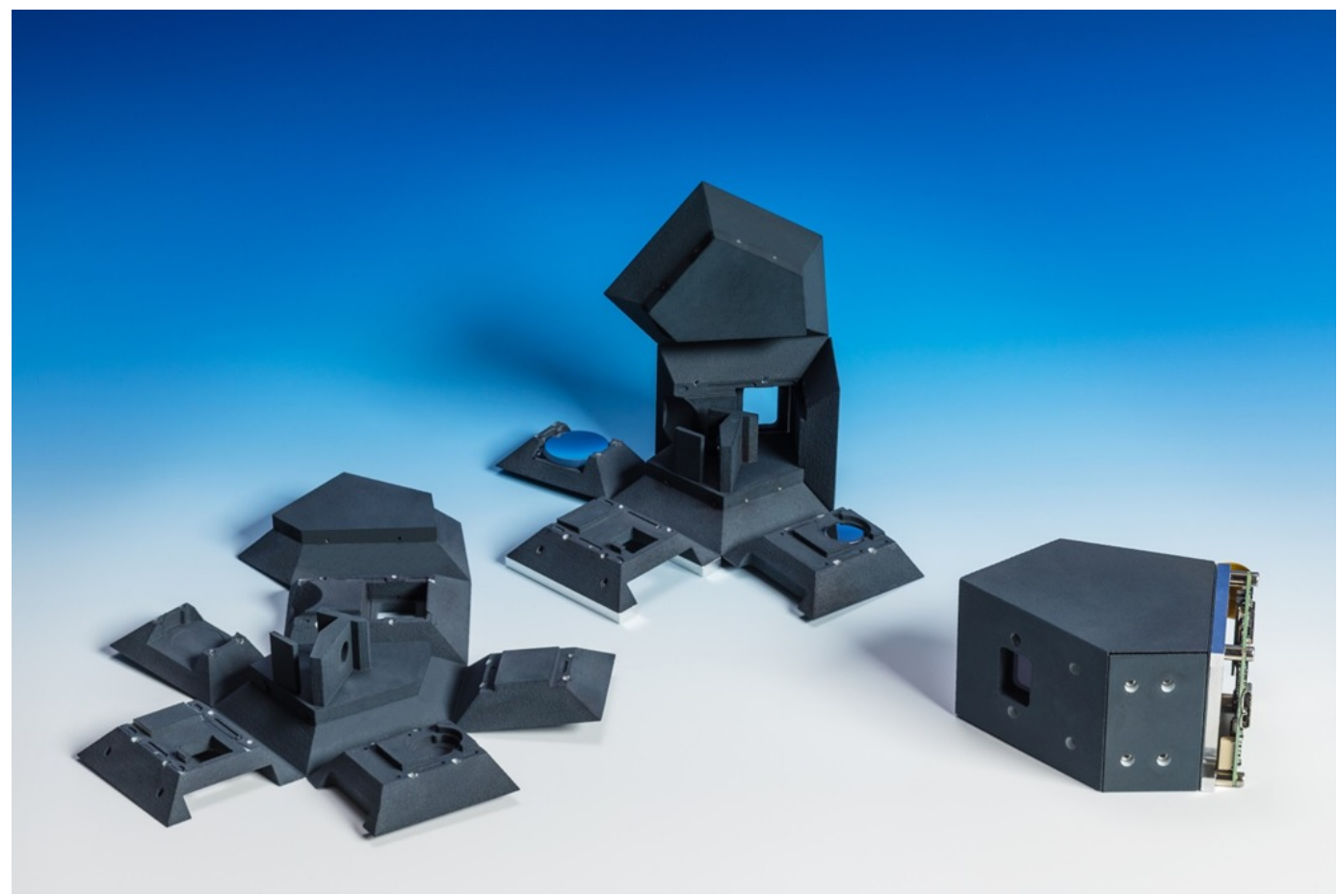

Figure 6. 3d printed substrate, substrate with components, folded system (from left to right)

The system electronics were attached to the optical bench. Data was transferred to the host PC and stored. Images were taken and the quality was evaluated. The optical path was aligned well but in the first trial the focus was out of the specified range. After minor rework of the substrate the focus was adjusted and the image quality was in a range as expected for this simple device (fig. 7).

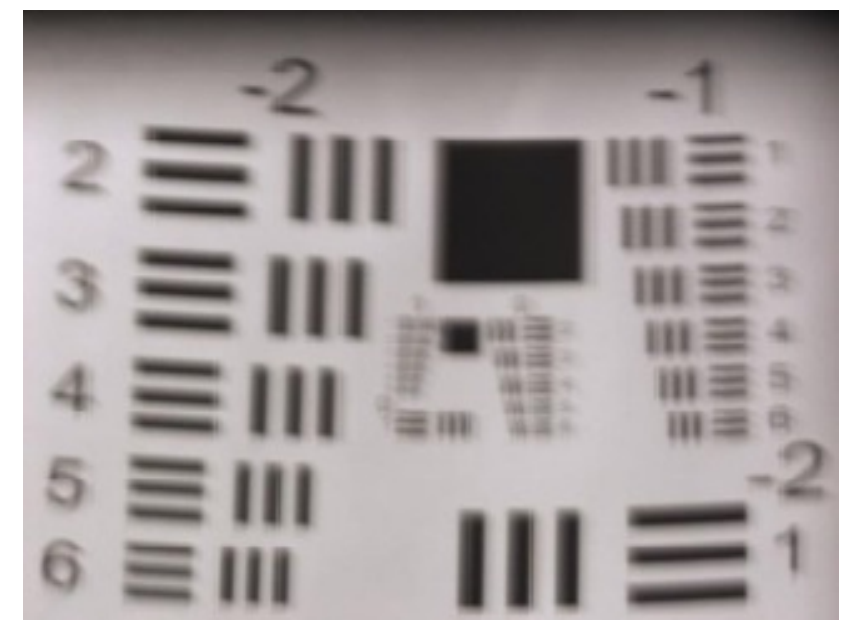

Figure 7. Image taken with a completely reflective camera built by "place and bend assembly" 


\section{CONCLUSIONS}

A new approach to realize optical systems especially off-axis designs has been presented. A planar substrate featuring preprocessed bending lines is applied for the placement of the components by conventional planar technologies. Afterward the substrate is bent to a 3D body and the optical path inside forms automatically. The so called "place and bent assembly" offers option for further miniaturization and cost efficient production of complex optical systems.

For test issues a 3D printed substrate was used to realize a simple design for a completely reflective camera device. The entrance window, two spherical mirrors, an aperture stop and the detector were assembled applying a conventional planar tool. The substrate was bent and fixed. After attaching the system electronics test images were taken and evaluated successfully. Thus the working principle of "place and bent assembly" was proven.

\section{OUTLOOK AND FUTURE APPLICATIONS}

The new approach will enable the integration of complex off axis optical systems in a very small outline offering options for low cost mass production. This will open applications with extended or broadband spectral range. Additional information about chemical composition or thermal signature can be accessed. Topics like food analysis, fitness and health or medical can be based on systems like near infrared spectrometers for mobile phones [15]. Multispectral cameras for autonomous driving vehicles may be shrank to fit into the base of the inside rearview mirror.

The next step within the ongoing development will be design and simulation of a spectrometer device starting with the sugar cube prototype design.

\section{Acknowledgements}

The authors would like to thank Mr. Jens Kruse for his engagement within this research work. The recent development is supported by Fraunhofer MEF program under grant contract no. 600890.

\section{REFERENCES}

[1] Patent DE102016221303.2

[2] Photograph published in online issue of Badische Zeitung from August, $1^{\text {st }} 2017$

[3] A. Kutter, "Der Schiefspiegler. Ein Spiegelteleskop für hohe Bilddefinition", Biberach Fritz Wichardt, 1953

[4] K. Seidl, J. Knobbe, D. Schneider, H. Lakner, "Distortion correction of all-reflective unobscured optical-power zoom objective", Appl. Opt. 49, 2712-2719 (2010)

[5] H. Schenk et al. „A new driving principle for micromechanical torsional actuators“ Micro Electro Mechanical Systems 1999, New York/USA, The American Society of Mechanical Engineers ASME (1999), 333-338

[6] M. Scholles, A. Bräuer, K. Frommhagen, Ch. Gerwig, B. Höfer, H. Lakner, H. Schenk, M. Schwarzenberg, „Design of miniaturized optoelectronic systems using resonant micro scanning mirrors for projection of full-color images", Proc. of SPIE Vol. 6288, 628807-1 - 628807-12 (2006)

[7] F. Woittennek, J. Knobbe, T. Pügner, H. G. Dallmann, U. Schelinski, H. Grüger, "MEMS scanner mirror based system for retina scanning and in eye projection", Proc. of SPIE Vol. 9375 - 5 (2015) 
[8] F. Zimmer, H. Grüger, A. Heberer, T. Sandner, H. Schenk, H. Lakner, A. Kenda, W.Scherf „Development of high-efficient NIR-scanning gratings for spectroscopic applications" SPIE 6114_9, (2006)

[9] H. Grüger, A. Wolter, T. Schuster, H. Schenk, H. Lakner, "Realization of a spectrometer with micromachined scanning grating" SPIE 4945, 46-53, (2003)

[10] H. Grüger, T. Pügner, J. Knobbe, H. Schenk, „First application close measurements applying the new hybrid integrated MEMS spectrometer", Proc. of SPIE Vol. 8726 872609-1 - 872609-09 (2013)

[11] T. Egloff, H. Grüger, F. Zimmer, H. Schenk, M. Scholles, H. Lakner, "NIR Hyperspectral Imaging using MOEMS scanning grating chip and linear detector array", Proc. of SPIE Vol. 6765-18 (2007)

[12] K. Seidl, J. Knobbe, H. Grüger, "Design of an all-reflective unobscured optical-power zoom objective", Appl. Opt. 48, 4097-4107 (2009)

[13] H. Grüger, „All-reflective optical power zoom objectives”, Proc. of SPIE Vol. 9580, 95800H-1 95800H-13 (2015)

[14] US Patent 8,045,159 B2

[15] T. Pügner, J. Knobbe, H. Grüger, "Near-Infrared Grating Spectrometer for Mobile Phone Applications", Applied Spectroscopy 2016, Vol. 70(5) 734-745 (2016) 